\title{
Contenidos de isómeros trans de los ácidos grasos en productos cárnicos. (I) Embutidos.
}

\author{
Por N. Hernández, R. Codony, M. Rafecas y J. Boatella
}

\author{
Departamento de Ciencias Fisiológicas Humanas y de la Nutrición. Nutrición y Bromatología. \\ División de Ciencias de la Salud. Universidad de Barcelona. \\ * Este trabajo ha sido subvencionado por la "Comissió Interdepartamental de la Recerca i \\ Innovació Tecnológica" (C.I.R.I.T.). Generalitat de Catalunya.
}

\section{RESUMEN}

Contenidos de isómeros trans de los ácidos grasos en productos cárnicos. (l) Embutidos.

En este trabajo se determinan los contenidos de isómeros trans de la fracción de los ácidos grasos, en muestras de embutidos diversos (total 48 muestras), mediante extracción previa de la fracción grasa y posterior análisis por cromatografia de gases capilar, con columna $\mathrm{CP}$ Sil 88. Los resultados muestran una gran variabilidad, entre 0,14 y $1,63 \%$ de C18: 1 t y entre 0,37 y $2,14 \%$ de formas trans totales.

PALABRAS-CLAVE: Acido graso - Embutido cárnico - Isómero trans (contenido).

\section{SUMMARY}

Trans isomers contents of the fatty acids in meat products. (I) Sausages.

We have studied the trans isomers contents of the fatty acids fraction in several types of Spanish sausages, after a previous fat extraction and gas chromatography determination using CP Sil 88 coated capillary columns. The results obtained show a great variability: from 0,14 to $1,63 \%$ for $\mathrm{C} 18: 1 \mathrm{t}$ and from 0,37 to $2,14 \%$ for the total trans fatty acids content.

KEY-WORDS: Fatty acid - Meat sausage - Trans isomer (content)

\section{INTRODUCCION}

El interés del estudio de las llamadas formas isoméricas de los ácidos grasos en productos cárnicos se debe, independientemente de sus posibles implicaciones nutricionales, (12), (3), (20), a que su presencia en estos substratos puede tener dos orígenes. En primer lugar estos compuestos pueden formarse en el estómago de los rumiantes, donde la flora propia hace que actúen isomerasas y reductasas sobre los ácidos insaturados ingeridos que conducen a la formación de, entre otros, el llamado ácido vaccénico (C18: 1, 11-trans), (6), (2), (9). En consecuencia, pueden encontrarse estas formas en productos derivados, tales como leche, carne, sebos, embutidos, quesos, etc. Según Bucharles et al., las grasas de los rumiantes pueden contener entre un 5 y un $7 \%$ de isómeros trans (4), formando una fracción compleja pero que, no obstante, brinda notables posibilidades en el control y tipificación de este tipo de productos (19).

Por otra parte, a través de las grasas utilizadas en la alimentación animal, pueden aparecer en mayor o menor grado formas isoméricas originadas en los tratamientos a que éstas han sido sometidas (refinación, hidrogenación, calentamientos, etc.), (15), (11), (13), (5), (10).

Estos isómeros, ya sean de posición o de configuración, aparecerán después en la grasa del animal. Es por ello que en los productos derivados del cerdo, su presencia se deberá fundamentalmente a los niveles de estas formas isoméricas presentes en su alimentación.

En relación con todo ello, estudios realizados por Slover et al. (16) en los lípidos que contiene la carne de buey, encuentran un porcentaje de monoenos trans (C18: 1-trans totales) que oscilan entre 1, 4 y $4,4 \%$, según el contenido graso del tejido muscular, y pequeñas cantidades de isómeros trans del ácido linoléico (C18:2, 9t,12t y $C 18: 2,9 c, 12 t)$, del orden del $0,2 \%$. Estos mismos autores (17), al estudiar la grasa de cerdo encuentran lógicamente, cantidades mucho menores de isómeros trans, un $0,2 \%$ de trans monoenos y un $0,014 \%$ de $\mathrm{C} 18$ : $2 \mathrm{c}, \mathrm{t}$.

El objetivo de este trabajo es pues el de evaluar la presencia de estas formas isoméricas en productos cárnicos y en concreto en diferentes tipos de embutidos, con objeto de conocer los contenidos de isómeros trans, las desviaciones existentes y el estudio de la variabilidad.

\section{MATERIALES $Y$ METODOS}

\subsection{Muestras}

Para este estudio se han utilizado muestras de salchichón, fuet y de chorizo como representantes del 
grupo de embutidos crudos y curados, así como de la butifarra catalana como ejemplo de los productos cocidos. El total de muestras analizadas ha sido de 48, distribuidas de la siguiente forma:

- Salchichón y fuet (26)

- Chorizo (14)

- Butifarra catalana (8)

Las muestras fueron suministradas por los gre- mios de charcuteros de Barcelona, Gerona, Lérida y Tarragona y fueron seleccionadas por ellos entre sus afiliados, de modo que quedaran representados los principales elaboradores. A todas las muestras se les exigía su pertenencia a la calidad "extra". De cada muestra se tomaron dos alícuotas que se analizaron paralelamente, siendo los resultados reseñados el valor medio de ambos análisis. Todos los embutidos se mantuvieron en refrigerador y envasados al vacío hasta

Tabla 1

Descripción y composición de las muestras: Humedad, grasa y composición en ácidos grasos.

\begin{tabular}{|c|c|c|c|c|c|c|}
\hline Muestra no & Tipo & $\% \mathrm{H} 2 \mathrm{O}$ & $\%$ GRASA & AGS & AGFI & A.GMI \\
\hline 1 & $\mathbf{S}$ & 26,80 & 28,48 & 34,27 & 26,14 & 46,28 \\
\hline 2 & $\mathrm{~s}$ & 41,11 & 35,04 & 40,90 & s. 45 & 45,26 \\
\hline 3 & $\mathrm{~s}$ & 27,19 & 34,14 & 45,71 & 9,83 & 46,23 \\
\hline 4 & $\mathrm{~s}$ & 17,90 & 46,87 & 39,72 & 11,36 & 45,87 \\
\hline 5 & $\mathbf{S}$ & 31,25 & 34,74 & 36,01 & 12,02 & 46,74 \\
\hline 6 & $\mathrm{~s}$ & 26,97 & 36,78 & 37,90 & 11,94 & $47,4 \epsilon$ \\
\hline 7 & $\mathbf{S}$ & 36,08 & 13,86 & 36,04 & 11,83 & $4 \varepsilon, 04$ \\
\hline 8 & $\mathrm{~S}$ & 32,49 & 40,60 & 38,23 & 13,01 & $45, \in \varepsilon$ \\
\hline s & $\mathrm{s}$ & 38,96 & 40,96 & 36,54 & 24,29 & 45,52 \\
\hline 10 & $\mathrm{~s}$ & 29,12 & 61,45 & 37,11 & $13,5 \varepsilon$ & $46, \in 7$ \\
\hline 11 & $\mathrm{~s}$ & 29,89 & 64,28 & 35,35 & 14,52 & 46,01 \\
\hline 22 & $\mathrm{~s}$ & 23,45 & 71,15 & 34,73 & 14,56 & 47,34 \\
\hline 13 & S & 27,89 & 51,75 & 34,44 & 14,83 & 46,90 \\
\hline 14 & S & 31,40 & 49,29 & 34,99 & $12, \varepsilon 1$ & 48,42 \\
\hline 15 & $\mathrm{~S}$ & $26, \in 0$ & 48,42 & 37,85 & 10,52 & $4 \varepsilon, 46$ \\
\hline $1 \epsilon$ & $S$ & 34,48 & 47,33 & $3 \in, 07$ & $1=50$ & 47,87 \\
\hline 17 & $\mathrm{~S}$ & 24,88 & $48, \sigma \epsilon$ & 38,17 & 11,83 & $4 \in, 45$ \\
\hline 18 & $E$ & 39,00 & $37, \varepsilon 7$ & 34,56 & 14,15 & $43, c \cdot \varepsilon$ \\
\hline 19 & E & 31,50 & 40,15 & 37,38 & 11,49 & 48,55 \\
\hline 26 & F & 30,50 & 51,05 & 38,77 & 8,26 & $50,0 E$ \\
\hline 21 & $\mathbf{F}$ & 24,52 & 47,55 & 30,74 & 11,93 & 49,47 \\
\hline $2 \bar{c}$ & F & $5 ?, 05$ & 40,27 & 44,07 & 11,34 & $4 C, 44$ \\
\hline 23 & $E$ & 32,47 & 35,75 & 37,14 & 9,81 & $49, \equiv 8$ \\
\hline 24 & F & $22,8 \in$ & 42,97 & 37,87 & 13,27 & 45,55 \\
\hline 25 & F & 29,40 & 47,97 & 33,72 & 16,15 & $45: 54$ \\
\hline$\because \in$ & $E$ & 19,10 & $\leq 7,85$ & 27,06 & 12,51 & $46, ? \div$ \\
\hline 27 & $\mathrm{CH}$ & 24,10 & $=8,97$ & $\Xi 5,24$ & 14.87 & $\leq 6, \Xi 2$ \\
\hline 28 & $\mathrm{CH}$ & 39,70 & 38,96 & $34,0=$ & $I \in, 77$ & $\leq 7,1 \subseteq$ \\
\hline 29 & $\mathrm{CH}$ & 46,12 & 48,43 & 33,55 & 15,77 & 47,76 \\
\hline 30 & $\mathrm{CH}$ & 57,50 & $\equiv 5,39$ & 37,88 & 15,32 & $4 \equiv, 54$ \\
\hline $3:$ & $\mathrm{CH}$ & $2 \epsilon, 30$ & 44,37 & 35,31 & 13,27 & $\triangle \varepsilon, \subseteq 7$ \\
\hline 32 & $\mathrm{CH}$ & $36, \varepsilon 0$ & 54,65 & 35,30 & 12,53 & $4 \in, \Omega \varepsilon$ \\
\hline 23 & C: & 27,94 & 69,40 & 36,53 & 12,45 & 47,41 \\
\hline 34 & $\mathrm{CH}$ & 42,90 & 71.75 & 35,64 & 12,95 & 49,01 \\
\hline 35 & $\mathrm{CH}$ & 23,53 & 53,54 & 37,24 & 10,41 & 48,38 \\
\hline 36 & $\mathrm{CH}$ & 52,00 & 64,17 & $3 \epsilon, 40$ & 11,79 & 48,67 \\
\hline 37 & $\mathrm{CH}$ & 45,65 & 51,66 & 35,87 & 11,97 & $q \varepsilon, \in \in$ \\
\hline 38 & $\mathrm{CH}$ & 44,65 & 74,44 & 28,38 & 11,12 & $4 E, 70$ \\
\hline 39 & $\mathrm{CH}$ & 29,76 & 82,06 & 36,44 & 14,69 & 45.47 \\
\hline 40 & $\mathrm{CH}$ & 43,28 & 37,73 & 34,33 & 12,86 & 45,32 \\
\hline 41 & C & 74,08 & 7,37 & 37,45 & 12,98 & 45,95 \\
\hline 42 & $C$ & 78,03 & 1,90 & 35,56 & 11,92 & 47,00 \\
\hline 43 & $C$ & $7 c, 70$ & 9,06 & 35,49 & 12,52 & 48,53 \\
\hline 44 & $C$ & 77,40 & 19,00 & 34,06 & 14,93 & 47,50 \\
\hline 45 & $\mathrm{C}$ & $8 E, 44$ & 24,25 & 35,47 & 14,48 & 45,50 \\
\hline 46 & $C$ & 81,80 & 14,66 & 36,69 & 13,45 & 45.20 \\
\hline 47 & C & 74,11 & 4.60 & 37,05 & 14,29 & 43,30 \\
\hline $4 \varepsilon$ & $c$ & 7424 & 400 & 33,69 & 1107 & 50,0 \\
\hline
\end{tabular}

Tipo: S: Salchichón; E: Fuet; CH: Chorizc; C: Butifarra Catalana.

rGrasa: (sobre materia seca)

AGS: $\Sigma(\% C 16: 0+\%$ C18:0) sobre grasa total

AGPI: $\Sigma(\% C 18: 2 c c+i C 18: 2 c t+\% C 18: 2 t c+\% C 18: 2 t t+\% C 18: 3)$ sobre g.t.

AGMI: $\Sigma(\$ C 16: 1 c+? C 16: 1 t+2 C 18: 1 z+q C 18: 1 t)$ sobie g.t. 
el momento de la preparación del extracto graso, para lo cual ha sido necesario en todos los casos realizar una trituración previa que se ha llevado a cabo mediante el uso de una picadora.

\subsection{Métodos}

a) Obtención del pocentaje de humedad: Se ha llevado a cabo por desecación de un peso exactamente conocido de muestra a $102^{\circ} \mathrm{C}+2^{\circ} \mathrm{C}$. (ISO R1442) (1).

b) Preparación del extracto graso: Se ha utilizado el método propuesto por Folch et al. (8) porque se trata de un método poco agresivo, de interés en este caso, para evitar la formación de artefactos. Este método realiza dos extracciones con metanol: cloroformo (1:2) y posterior lavado con solución diluida de $\mathrm{CINa}$. Seguidamente se filtran las fases clorofórmicas obtenidas con $\mathrm{SO}_{4} \mathrm{Na}_{2}$ anhidro y finalmente se evapora el cloroformo mediante un evaporador rotatorio de vacío.

c) Determinación de ácidos grasos por cromatografía en fase gaseosa con columna capilar:

Preparación de los ésteres metílicos (14), (18). A una muestra de $190-200 \mathrm{mg}$ del extracto graso se adicionan, en un tubo de $16 \times 150 \mathrm{~mm}$ provisto de tapón de rosca, $2,5 \mathrm{ml}$ de metilato sódico $0,2 \mathrm{~N}$ y se calienta en un baño de agua hirviente durante 5-10 min, hasta la aparición de una sola fase. Enfriar y añadir $3,5 \mathrm{ml}$ de $\mathrm{BF}_{3}$ /metanol al $14 \%$ (Merck) y calentar en el baño de agua durante $15 \mathrm{~min}$. Después de enfriar, se adicionan $1 \mathrm{ml}$ de hexano y $2,5 \mathrm{ml}$ de solución saturada de $\mathrm{CINa}$, se agita vigorosamente durante $1 \mathrm{~min}$ y por último se transfiere la fase hexánica a un tubo que contiene sulfato sódico anhidro. Este método ha resultado especialmente adecuado para la protección de la columna cromatográfica utilizada, ya que trazas de humedad o de ácidos han demostrado ser muy perjudiciales para la fase estacionaria.

Analisis Cromatográfico: Se ha utilizado una columna capilar tipo WCOT Fused Silica, de fase líquida CP Sil 88, (Chrompack), de $50 \mathrm{~m}$ y $0,25 \mathrm{~mm}$ de diámetro interno, provista de un "retention gap" de $2 \mathrm{~m}$. El análisis se ha realizado a una temperatura del horno $190^{\circ} \mathrm{C}$ y con una relación de splitt de 1:100. Se ha utilizado un cromatógrafo Perkin-Elmer Sigma 2000, acoplado a un integrador HP3396A.

Para la identificación de los isómeros C18:1 y C18:2 se han utilizado estandars Merck y Sigma, así como los datos relacionados en la bibliografía (18) (5). Cabe señalar que con las condiciones cromatográficas descritas no se resuelven los isómeros de posición, por lo que los resultados se expresan como $\mathrm{C} 16: 1 \mathrm{t}, \mathrm{C} 18: 1 \mathrm{t}$ y $\mathrm{C} 18: 2 \mathrm{t}$ (cc, tc, ct, tt).

\section{RESULTADOS Y DISCUSION}

En la Tabla I se exponen junto a la descripción del producto, los valores de $\%$ de humedad y los de grasa total calculada en porcentaje de materia seca, así como los porcentajes totales de ácidos grasos saturados, poliinsaturados y monoinsaturados. En primer lugar y en relación con el contenido de humedad debe señalarse que los valores medios para cada grupo fueron de $30,2 \%$ (salchichón), 39\% (chorizo) y $77,5 \%$ (butifarra catalana), cifras que se ajustan a lo señalado en la Norma de Calidad, si bien debe destacarse que un $10,4 \%$ de las muestras, superaron el valor máximo. Para el contenido graso las cifras medias obtenidas fueron de $44,1 \%, 54,7 \%$ y $11,5 \%$ respectivamente, superando los valores máximos permitidos un $16,7 \%$ de las muestras.

Los valores globales correspondientes a los porcentajes de ácidos grasos (saturados, monoinsaturados y poliinsaturados) (Tabla II) son concordantes con los obtenidos por otros autores (7), (4), (16).

La Tabla III recoge los porcentajes de los ácidos grasos trans hallados así como el del total de formas trans. Los ácidos C18:1t se encuentran presentes en todas las muestras estudiadas, con valores que oscilan entre 0,15 y $1,34 \%$ ( $x: 0,41 \%$, Sn-1: 0,30, C.V.: 71,4$)$ para el salchichón y fuet, entre 0,14 y $1,63 \%$ ( $x: 0,63 \%$, Sn-1: 0,50 y C.V.: 76,8$)$ para el chorizo y entre 0,18 y $0,95 \%$ (x: $0,58 \%, S n-1: 0,26 \%$ y C.V.: 44,8 ) para butifarra catalana.

En relación con los contenidos de formas trans totales $(C 16: 1+C 18: 1+C 18: 2)$ debe señalarse una gran dispersión de resultados, con unos valores máximos de $2,04 \%$ para salchichones y $2,14 \%$ para chorizos. Los valores medios obtenidos fueron de $0,82 \pm 0,40$

Tabla II

Valores medios, desviaciones estandar y coeficientes de variación de los tres grupos de muestras estudiadas, para los totales de AG Saturados, Poliinsaturados y Monoinsaturados.

\begin{tabular}{cccccccccc} 
& \multicolumn{3}{c}{ AG Saturados } & \multicolumn{2}{c}{ AG Polinsaturados } & \multicolumn{3}{c}{ AG Monoinsaturados } \\
Tipo & $\mathrm{x}$ & Sn-1 & C.V. & $\mathrm{x}$ & Sn-1 & C.V. & $\mathrm{x}$ & Sn-1 & C.V. \\
$\mathrm{S}+\mathrm{F}$ & $3.7,14$ & 2,31 & 6,2 & 12,45 & 1,99 & 16,0 & 46,92 & 1,90 & 4,0 \\
$\mathrm{CH}$ & 35,94 & 1,40 & 3,9 & 13,34 & 1,87 & 14,0 & 47,39 & 1,58 & 3,3 \\
C & 35,96 & 1,16 & 3,2 & 13,51 & 1,11 & 8,2 & 46,15 & 1,71 & 3,7
\end{tabular}


Tabla III

Porcentajes relativos de los ácidos grasos trans, en los embutidos analizados.

\begin{tabular}{|c|c|c|c|}
\hline Muestra ne & C16:1t & C18:1t & C18:2ct \\
\hline 1 & $0,42 \ldots$ & 0,44 & 0,010 \\
\hline 2 & - & 0,44 & 0,002 \\
\hline 3 & 0,33 & 0.45 & 0,0104 \\
\hline 4 & 0,28 & 0,26 & 0,002 \\
\hline 5 & 0,32 & 0.25 & 0.020 \\
\hline 6 & 0,37 & 0,44 & 0,010 \\
\hline$?$ & 0,52 & 0,78 & 0,003 \\
\hline 8 & 0,34 & 0,15 & 0,006 \\
\hline 9 & 0,43 & 0,20 & 0,002 \\
\hline 10 & 0,27 & 0,24 & $c, 003$ \\
\hline 11 & 0,36 & 0.30 & 0.010 \\
\hline 12 & 0,32 & 0,14 & 0,005 \\
\hline 13 & $(1,34$ & 0,26 & $c, 003$ \\
\hline 14 & $0, \in I$ & 1,013 & 0,007 \\
\hline 15 & $C, 34$ & 0,19 & 0,001 \\
\hline 20 & 0,67 & 0,32 & 0,010 \\
\hline 27 & 0.33 & 0,73 & 0,020 \\
\hline $1 \varepsilon$ & 0,35 & $0,2 \varepsilon$ & 0,004 \\
\hline 10 & 0,18 & 0,20 & 0,010 \\
\hline 20 & 0,37 & 0,18 & 0,010 \\
\hline 22 & 0,34 & 0,21 & 0,020 \\
\hline 22 & 0,44 & $0,9 \vdots$ & $0.0=0$ \\
\hline 23 & $c, 34$ & $0,2 z$ & - \\
\hline 24 & 0,39 & 0,53 & 0,003 \\
\hline 25 & 0,41 & 0,24 & 0,010 \\
\hline$\Delta \epsilon$ & 0,69 & 1,34 & 0.010 \\
\hline 27 & 0.38 & 0,37 & 0,007 \\
\hline 28 & 0,32 & 0,22 & $0,00 \geq$ \\
\hline 23 & $c,=4$ & $0,9 \varepsilon$ & 0,010 \\
\hline$\equiv 0$ & 0,38 & 0,78 & 0,110 \\
\hline 31 & 0,33 & $0,4 i$ & 0,010 \\
\hline 32 & 0,49 & 1,53 & 0,003 \\
\hline 33 & 0,61 & 0,92 & 0,003 \\
\hline 34 & 0,16 & 0,31 & 0,006 \\
\hline 35 & 0,25 & 0,23 & $c, 004$ \\
\hline 36 & 0.61 & 1,50 & - \\
\hline 37 & 0,56 & 0,98 & 0,001 \\
\hline 38 & 0,23 & 0,24 & 0,010 \\
\hline 39 & $0,2 \epsilon$ & 0,17 & 0,010 \\
\hline 40 & 0,38 & 0,14 & 0,004 \\
\hline 41 & 0,38 & 0,18 & 0,008 \\
\hline 42 & 0,40 & 0,88 & 0,002 \\
\hline 43 & 0,58 & 0,78 & 0,003 \\
\hline 44 & 0,43 & 0,51 & 0,020 \\
\hline 45 & 0,45 & 0,45 & 0,020 \\
\hline 46 & 0,28 & 0,43 & 0,010 \\
\hline 47 & 0,35 & 0.47 & 0,003 \\
\hline 48 & 0,51 & 0,95 & 0,002 \\
\hline
\end{tabular}

$c 18: 2 t c$
0,010
0,005
0,004
0,001
0,010
0,007
0,020
0,004
0,010
0,003
0,008
0,005
0,010
0,002
0,020
0,0
0,006
0,010
0,002
0,004
0,040
0,010
0,0
0,040
0,003
0,001
0,010
0,0
0,010
0,080
0,020
0,005
0,002
0,000
0,000
0,007
0,002
0,040
0,001
0,020
0,007
0,020
0,040
0,005
0,010

0

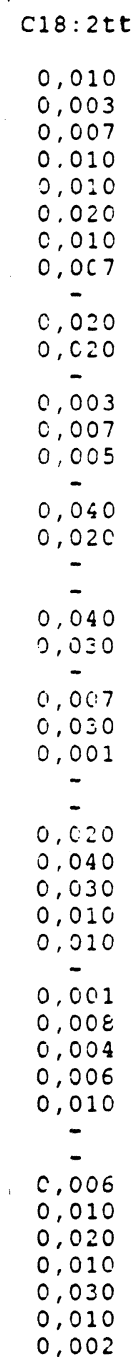

T.Trans
0,39
0,45
0,79
0,55
0,61
0,85
1,33
0,51
0,73
0,54
0,70
0,47
0,62
1,66
0,56
1,00
1,10
0,67
0,37
0,50
0,65
1,40
0,57
0,98
0,69
2,04
0,77
0,54
1,26
1,39
0,80
2,14
1,55
0,49
0,49
2,11
1,55
0,49
0,49
0,53
0,58
1,29
1,39
1,02
0,93
0,89
0

(C.V. $=48,8)$ para el salchichón, $1,04 \pm 0,62$ (C. $V .=59,6)$ para el chorizo y de $1,04 \pm 0,31$ (C. $V .=29,8$ ) para la butifarra catalana. Estas cifras son marcadamente inferiores a las señaladas en la bibliografía (14), (15). Estos niveles y su elevada variabilidad, comparada con la de los ácidos grasos característicos de esta grasa, demuestran la influencia de los distintos tipos de grasa utilizadas en alimentación animal.

Por lo que respecta a los trans monoenos se observa una correlación estadísticamente significativa (texp. $=5,544 ;$ ttab. $=3,520 ;$ para $p=0,001$ ) entre $C 16: 1$ y $C 18: 1$, lo que parece indicar que ambos compuestos poseen un mismo origen, fundamentalente la grasa de la alimentación.

Por otra parte, un análisis de la varianza de los valores correspondientes a los contenidos en ácidos trans, para los tres tipos de productos estudiados, revela que no existen diferencias estadísticamente significativas entre ellos y también los estudios de correlación realizados demuestran que no existe relación entre el contenido graso y la cantidad total de formas trans. 
1. Análisis de Alimentos.-Métodos Oficiales y Recomendados por el Centro de Investigación y Control de Calidad.-Ministerio de Sanidad y Consumo, 1985.

2. Brisson, G.-"Lipides et nutrition humane".-Les Presses de I'Université Laval. Masson, Paris, 1982.

3. The British Nutrition Foundation.-"Trans Fatty Acids".-The report of the B.N.F. Task Force. London, 1987.

4. Bucharles, C.; Gerardot, L.; Girard, J.P., y Denoyer, C.-“Les lipides animaux dans la filière viande".-Volumen I. "Nomenclature, éléments de métabolisme, facteurs de variation pour les espèces couramment consommées en France".-Ed. A.P.R.I.A. Paris, 1985.

5. Dutton, J.-"Hidrogenation of fats and its significance" in "Geometrical and Positional Fatty Acid Isomers".-Chap. 1, p. 3.-E.A Emken and H.J. Dutton (Eds).-Amer. Oil Chem. Soc. Champaign (Illinois), 1979.

6. Entressangles, B.-"Mise au point sur les isoméres trans alimentaires".-Rev. Fr. Corps Gras. 33 (1986) 47-58.

7. Feinberg, M.; Favier, J.C., y Ireland, J.-"Ripert Répertoire géneral des aliments".-Tome I. "Table de Composition des Corps Gras"--I.N.R.A., Paris, 1987.

8. Folch, J.; Lees, M., y Sloane Stanley, G.H.-"A simple method for the isolation and purification of total lipids from animal tissues".-J. Biol. Chem. 226 (1957) 497-509

9. Gunstone, F., y Norris, F.A.-"Lipids in Foods. Chemistry. Biochemistry and Technology".-Pergamon Press, 1983.

10. Hernández, N., y Boatella, J.-"Contenidos de isómeros trans de los ácidos grasos en margarinas":-Grasas y Aceites 39 (1988) 348-352.

11. Koritala, S.-“Selective hydrogenation with copper catalyst: V Kinetics and mechanism at high pressure".-J. Am. Oil Chemists' Soc. 57 (1980) 293-298.

12. Kummerow, F.A.-"Dietary effects of trans fatty acids".-J. Enviromental Patol. Toxicol. 6 (1986) 123-149.

13. Mallet, G.; Dimitriades, C., y Ucciani, E.-"Recherches sur les mecanismes de l'hidrogénation sélective: il-Structure des isómeres formés par hydrogénation d'un modéle simulant l'huile de colza".-Rev. Fr. Corps Gras 32 (1985) 387-395.

14. Metcalfe, L.D.; Schmitz, A.A., y Pelka, J.R.-"Rapid preparation of fatty acid esters from lipids for gas chromatographic analysis".-Anal. Chem. 38 (1966) 514-515.

15. Sebedio, J.L.; Grandgirard, A., y Prevost, J.-"Linoleic acid isomers in heat treated sunflower oils".-J. Am. Oil Chemists' Soc. 65 (1988) 362-366.

16. Slover, H.T.; Lanza, E.; Thompson, R.H.; Davis, C.S., y Merola, G.V.-"Lipids in raw and cooked beef".-J. Food Composition and Analysis 1 (1987) 26-37.

17. Slover, H.T.; Thomson, R.H.; Davis, C.S., y Merola, G.V.-"The lipid composition of raw and cooked fresh pork".-J. Food Composition and Analysis 1 (1987) 38-52.

18. Slover, H.T., y Lanza, E.-"Quantitative analysis of food fatty acids by capillary gas chromatography".-J. Am. Oil Chemists' Soc. 56 (1979) 933-943.

19. Wolff, J.P.-"Manuel d'analyse des corps gras".-Ed. Azoulay, Paris (1968) 377-382.

20. Zevenbergen, J.L.-“Biological effects of trans fatty acids".-in C. Galli, E. Feli Fat Production and Consumption Technologies and Nutritional Implications. Vol. 131. Series A: Life Sciences. Plenum Press, 1986.

(Recibido: Julio 1990) 